\title{
Tissue engineering is a promising method for the repair of spinal cord injuries (Review)
}

\author{
WENCHEN JI ${ }^{1,2}$, SHOUYE HU ${ }^{1}$, JIAO ZHOU ${ }^{3}$, GANG WANG $^{1}$, KUNZHENG WANG $^{1}$ and YUELIN ZHANG ${ }^{1}$ \\ ${ }^{1}$ Medical School of Xi'an Jiaotong University, Xi'an, Shaanxi 710061, P.R. China; ${ }^{2}$ Department of Physiology, \\ College of Medicine, University of Sydney, Sydney 2006, Australia; ${ }^{3}$ Department of Surgery, \\ The Third Affiliated Hospital, Medical School of Xi'an Jiaotong University, \\ Xi'an, Shaanxi 710068, P.R. China
}

Received July 30, 2013; Accepted December 10, 2013

DOI: $10.3892 /$ etm.2013.1454

\begin{abstract}
Spinal cord injury (SCI) may lead to a devastating and permanent loss of neurological function, which may place a great economic burden on the family of the patient and society. Methods for reducing the death of neuronal cells, inhibiting immune and inflammatory reactions, and promoting the growth of axons in order to build up synapses with the target cells are the focus of current research. Target cells are located in the damaged spinal cord which create a connect with the scaffold. As tissue engineering technology is developed for use in a variety of different areas, particularly the biomedical field, a clear understanding of the mechanisms of tissue engineering is important. This review establishes how this technology may be used in basic experiments with regard to SCI and considers its potential future clinical use.
\end{abstract}

\section{Contents}

1. Introduction

2. Seed cell

3. Scaffold

4. Growth factor

5. Conclusion

\section{Introduction.}

Spinal cord injury (SCI) typically results in a permanent disability, which may be an economic burden on the family of the patient and society, and to date there is no effective method

Correspondence to: Professor Yuelin Zhang, Department of Neurosurgery, The Third Affiliated Hospital, Medical School of Xi'an Jiaotong University, 76 Yanta West Road, Xi'an, Shaanxi 710061, P.R.China

E-mail: yuelinzhang1968@163.com

Key words: tissue engineering technology, spinal cord injury of treatment (1). Every year there are 12,000 new cases of SCI in the USA, with the total number of American individuals living with a SCI estimated at 259,000 (2). The development of a cure for SCI is a research topic of particular interest.

SCI triggers a series of pathological steps that include the original insult and subsequent secondary steps, such as ischemia, anoxia, free-radical formation and excitotoxicity (3). The original insult refers to the mechanical trauma that leads to the SCI. During this period, the spinal cord tissue is disrupted by an external force, produced by the original insult mechanism. The most recognized mechanism for injury includes the following steps $(4,5)$ : i) contusion of the spinal cord when injury occurs and ii) prolonged compression due to displacement of vertebral bony structures and tissues. Following the initial spinal cord trauma, secondary damage is apparent. The post-trauma inflammatory response is particularly significant and, through a series of complicated cellular and molecular interactions, plays a key role in the entire secondary phase following SCI $(6,7)$.

Clinically, the treatment of SCI mainly focuses on reducing secondary damage and the prevention of complications (8). However, if the aim is to successfully repair the SCI and promote functional recovery, the following must be achieved $(9,10)$ : i) reduction of the death of neuronal cells, ii) inhibition of glial scar formation, since glial scarring decreases axon growth, iii) provision of a matrix at the injury site to supply the nutrients required to support axonal growth, iv) elimination of immune reactions and v) facilitation of the build-up of functional synapses and the transmission of neurotransmitters by regenerating axons. The key to treatment is to establish axonal regeneration in the damaged area with the anticipation that it will extend through the damaged area to establish a connection with the target neurons in the spinal cord. To date, it has been demonstrated that blocking inhibitory molecules and antagonizing secondary injury mechanisms promotes axon growth, by using trophic factors, cellular transplants and polymeric scaffolds (11). However, no significant functional recovery has been observed; therefore, a novel method is required.

Tissue engineering is an emerging area in biomaterial research that possesses great therapeutic potential. However, in order for it to be used clinically there are challenges that need 
to be overcome (12). In recent years, studies have begun to explore the possibility of using tissue engineering technology to repair SCIs, specifically, by using seed cells, neurotropic factors and a biological scaffold (Fig. 1). The aim of this review is to discuss this tissue engineering method and investigate the hypothesis that, if a suitable seed cell, scaffold and growth factor are identified, tissue engineering offers bright prospects for future research and the potential for clinical use.

\section{Seed cell}

The basis for tissue engineering is the seed cell, which is also the bottleneck that has been restricting the development of tissue engineering. The main reason for this is that certain cells, such as cartilage cells and endothelial cells, are limited and it is not possible to construct a large organization through small quantities of tissues in vitro (13). In general, tissue engineering seed cells must meet the following criteria $(14,15)$ : i) successful ability to proliferation in vitro, ii) good cell viability and function, iii) high level of purity, iv) no rejection by the immune system and v) high safety. With the continuous development of tissue engineering, stem cells, including embryonic stem (ES) cells, and adult stem cells are gradually entering the seed cell field, with many advantages, including wide availability, a strong ability to proliferate, the ability to differentiate into a variety of cells and the ability to form the corresponding organization. At present there are clinical problems with using seed cells, the selection of a suitable seed cell may resolve this problem (16).

ES cells and neural stem cells are the most important types of stem cell that were used in the early stages of seed cell research $(17,18)$. Transplanting ES cells into the brain was shown to significantly improve neurological function in an animal model of Parkinson's disease (19). The reason for this success is that the cells survived and were differentiated into different cells, such as oligodendrocytes, astrocytes and neurons. However, this method has certain ethical issues and problems with regard to rejection reactions (20). In addition, the availability of neural stem cells is limited and, therefore, their widespread clinical use is not viable (21). With advances in stem cell research, mesenchymal stem cells (MSCs) extracted from bone marrow (BMSCs) have been shown to contain pluripotent precursor cells, which have the ability to differentiate into various types of brain cell (22). In vivo transplantation of BMSCs into the brain has established that they migrate throughout various brain regions where they undergo differentiation into cells with astrocytic and neuronal phenotypes (23). However, if BMSCs are to be used clinically, the extraction of bone marrow from patients is necessary, which is likely to result in patient trauma. Therefore, an increasing number of studies have suggested the use of adult stem cells, which have all the advantages of BMSCs, but without the need to induce trauma in the patients in order to extract them.

Muscle-derived stem cells (MPSCs) are adult stem cells, commonly used in tissue engineering. Alessandri et al (24), have demonstrated that adult human skeletal muscle includes a population of progenitor stem cells capable of generating cells of the same lineage and have suggested that MPSCs are ideal seed cells. Lavasani et al (25) and Wu et al (26) have shown that MPSCs have the ability to differentiate into various cell types

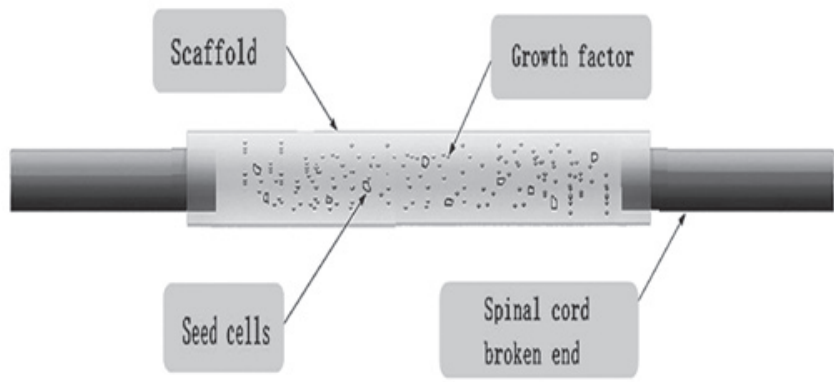

Figure 1. Scheme for the use tissue engineering technology in the repair of spinal cord injuries.

when placed within specialized inducing media. Danisovic et al $(27,28)$ focused on the immunological properties of MPSCs and hypothesized that their potential for differentiation may be useful in cell therapy for a variety of degenerative diseases. However, the majority of studies have investigated the use of MPSCs to cure coronary and urological diseases, with a limited number using MPSCs to repair nerve injuries. Woo et al (29) transplanted MDSCs into a cavernous nerve injury in a rat model, the result of which demonstrated that MDSCs were capable of improving erectile function. Stulpinas et al (30) and Shibuya et al (31) investigated the potential use of MPSCs in the treatment of non-acute myocardial infarction, with a swifter recovery. Kwon et al $(32,33)$ found that although MPSCs are a good seed cell, capable of differentiating into numerous cell types, the neural differentiation capacity of MDSCs is less than that of adipose-derived stem cells (ADSCs). Therefore, ADSCs may be the most suitable type of adult stem cell for use as seed cells in SCI treatment.

Adipose tissue is abundant in the body. Zuk (34) extracted cells from adipose tissue and observed that the morphology, biological characteristics and immune phenotypes of the cells were similar to those of BMSCs. An advantage of using ADSCs is that obtaining these cells is minimally invasive to the patient. If different types of induction medium are used, the cells may differentiate into adipocytes, osteoblasts, chondrocytes and neurons, which are the most common types of seed cells currently used in research, and represent a promising tool for SCI treatment (35-38). Recently, studies have shown that due to the secretion of various growth factors, such as hepatocyte growth factor (HGF), tumor necrosis factor- $\alpha$ (TNF- $\alpha)$, vascular endothelial growth factor (VEGF), brain-derived neurotropic factor (BDNF) and nerve growth factor (NGF), ADSCs may be used in the acute stages of SCI and have the potential to improve functional recovery, tissue preservation and neuronal regeneration (39-41). Oh et al (42) used ADSCs to treat SCI and observed functional improvements. Similar results were demonstrated by Barriga et al (43), Ferrero-Gutierrez et al (44) and Chung et al (45). Therefore, this type of stem cell may be considered to be the most suitable for use as a seed cell for the treatment of SCI.

\section{Scaffold}

Maintaining cell growth is challenging if the cells are injected directly into the damaged area. Opening the meninges may lead to cell loss, which is likely to inhibit the ability of the cells 
to adhere to the damaged tissues and proliferate. Therefore, in addition to seed cells, tissue-engineering scaffolds are also important and their potential use in the repair of the spinal has been the subject of study for several years (46).

The requirements of a scaffold for spinal cord regeneration are as follows $(47,48)$ : i) good biocompatibility, in order to avoid reactions with the immune system, ii) an ideal degradation rate and the formation of non-toxic degradation products, and iii) mechanical properties that are suitable for cell adhesion and axonal regrowth. There have been several studies that have concentrated on the microstructural design of porous scaffolds, which must be conditioned in vivo prior to implantation (49,50). However, this has the major disadvantage of increasing the difficulty of the design at the engineering level and surgical implantation may also be challenging. The best solution is to create a scaffold with a simpler design, that is easier to transplant and is suitable for various types of injury $(51,52)$.

Silk fibroin (SF) is obtained by degumming silk and acts as a natural structural albumen without physiological activity. It mainly consists of three simple amino acids: glycine, alanine and serine, which account for $85 \%$ of the total protein. Furthermore, SF has excellent mechanical properties, good compatibility and induces only a slight inflammation reaction in vivo. SF has been used as a scaffold for the treatment of SCI in certain experiments, but the disadvantage of this material is that when it is dry it is particularly brittle and difficult to handle (53). Therefore, in order to overcome this shortcoming, another polymer, chitosan, is added to the SF formulation. Chitosan has been investigated for its biocompatibility, biodegradability and toxicity in tissue engineering for several years, despite the disadvantages that it degrades rapidly and has a high swelling property $(54,55)$. Therefore, a blend of both materials to make silk fibroin-chitosan (SFCS) may avoid the limitations of pure SF and CS. Furthermore, the blend has good mechanical properties and may be used as a scaffold material for the repair of SCIs $(56,57)$.

In addition to SFCS scaffolds derived from natural components, injectable scaffolds are also particularly important for tissue engineering, as they are capable of filling the damaged site and may be delivered using a minimally invasive method (58). This type of scaffold also possesses the ability to mold to irregularly shaped damaged sites. Comolli et al (59) used a poly(N-isopropylacrylamide)-co-poly(ethylene glycol) (PNIPAAm-PEG) injection scaffold, which provided the sustained release of BDNF and neurotrophin-3 (NT-3) for up to four weeks; the constant secretion of these growth factors was identified to be a positive factor in functional recovery. However, the majority of these scaffolds require gelation (crosslinking) in vivo, which may result in complications, either from unreacted monomers or excess reactant (60).

Further to the two previously discussed types of scaffold, other scaffolds have been used successfully in the treatment of SCI. Kang et al (61) used poly (D,L-lactide-co-glycolide) to successfully treat transected spinal cords in rats; a certain degree of nerve regeneration and functional recovery was observed. A study by Liu et al (62) using nanofibrous collagen nerve conduits demonstrated that this type of scaffold is capable of promoting neural fiber growth following SCI, and is also capable of inhibiting glial scar hyperplasia. Zhu et al (63) used nanofibrous scaffolds as a drug delivery vehicle for the treatment of SCI in rats, and observed significant improvements in hindlimb function after three weeks. Teng et al (64) studied the use of a poly(lactic-co-glycolic acid) (PLGA) scaffold to treat SCI in rats. The authors identified that corticospinal tract fibers permeated the epicenter of the injury to the caudal cord and that local GAP-43 expression was increased, which lead them to hypothesize that PLGA increases the possibility of recovery following SCI. However, in contrast to the findings of Teng et al, a study by Du et al (65) demonstrated that a gelatin sponge is more suitable than a PLGA scaffold for transplantation into the spinal cord to promote the recovery of SCI.

In order to successfully use tissue engineering to repair SCI, the selection of a suitable scaffold is particularly important. Compared with a single component scaffold, a mixed scaffold (comprising several ingredients) may be more successful as it may minimize the disadvantages of the single component scaffold and provide a scaffold with increased functionality. Furthermore, compared with synthetic scaffolds, scaffolds prepared from natural components may be more advantageous, as the reaction of the immune system and the inflammatory reaction is reduced following implantation into the body.

\section{Growth factor}

Neurotrophic factors play an important role in functional recovery following SCI, as they protect neuronal cells from apoptosis and promote axonal regeneration (66). Neurotrophic factors may be divided into neurotrophins, ciliary neurotropic factor, the glial cell line-derived neurotrophic factor family and other growth factors or cytokines (67-69). The most frequently used neurotrophic factors are NGF, NT-3 and BDNF. NGF was discovered in 1950 and, as a core factor in the regulation of peripheral innervations, was found to have an effect on the CNS (70). Allen et al (71) demonstrated that NGF has a promising future as a therapeutic option for neurodegeneration. Weishaupt et al $(72,73)$ extracted BDNF from a porcine brain and identified that it had a broad-spectrum effect on peripheral and central neurons, with the exception of the ciliary ganglion neurons, sensory neurons, hippocampal neurons, cerebellar neurons, motor neurons, cholinergic neurons of the basal forebrain and midbrain dopaminergic neurons. BDNF expresses its biological effects through the activation and binding of TrkB (74). Stokols et al (75) discovered that a BDNF-incorporated agarose scaffold implanted into the spinal cord of a rat resulted in the linear-fashioned growth of regenerating axons through the scaffold. NT-3, which may be generated by the cloning of a multifunctional NGF gene, not only maintains motor neurons, sympathetic neurons and dopaminergic neuron differentiation, but also maintains the survival of sympathetic and sensory neurons and promotes nerve outgrowth in vitro $(76,77)$. To date, NT-3 is considered to be the only gene to promote the growth of the corticospinal tract (CST) following SCI. The biological effect of NT-3 is produced by the binding and activation of TrkC; NT-3 also has effects on TrkA and TrkB, but these are weak $(78,79)$.

Neurotrophic factors may be applied by the following three methods: local injection, cerebrospinal fluid injection and gene modification (80). Of these methods, the most important is gene modification. Studies have shown that the direct 
injection of neurotrophins into the site of injury results in an improved functional recovery compared with that in a control group to which growth factor is not administered; however, due to several factors, such as concentration, time limitation, and a short half-life, this treatment was unable to fully meet the requirements for nerve regeneration $(81,82)$. Cerebrospinal fluid injection has certain disadvantages, including the fact that it is not possible to restrict the location of the neurotrophic factor to the injury site, and recovery is less successful compared with that achieved using other methods. Therefore, an increasing number of researchers are focusing on gene modification (79).

There are two methods of applying gene therapy for the treatment of SCI (83-85): the direct transfer of the gene into the target cells in the human body, and cell-mediated gene therapy. The latter method, which requires the target gene to be transferred into an appropriate transplant cell, the selection of cells with a high level of gene expression, and the transplantation of the cells into the target tissue, is the most commonly used. Researchers have used transgenic technology to modify fibroblast cells, muscle cells and Schwann cells, which are subsequently transplanted into the injured area. The genetically modified cells may continue to express nutritional factor, the purpose of which is to promote nerve regeneration. Gene transfer vectors are divided into viral vectors and non-viral vectors (86). Viral vectors include adenoviruses, retroviruses and chronic viruses; retroviruses and chronic viruses are of particular interest as their transfer into the host genome may lead to long-term expression (87-89); Morizono and Chen (89) compared the efficiencies of three types of virus for the transfection of ADSCs, and observed that the highest transfection efficiency was achieved with a chronic virus. When an exogenous gene was transferred by chronic virus carriers into adipose stem cells, which were then induced in vitro, detection of the gene remained possible following osteogenic and adipogenic differentiation. Therefore, the combination of stem cells and chronic virus carriers is currently being studied (89). However, the clinical application may be simpler if growth factors are slowly released from scaffolds, rather than being delivered by gene transfer, using biomaterials that are capable of providing the protracted release of loaded proteins.

\section{Conclusion}

Tissue engineering is a promising method that may be used for the treatment of SCI. It involves three factors: the seed cell, the scaffold and a growth factor. For the repair strategy to be successful, the selection of an appropriate seed cell, scaffold and growth factor is required. Considering the seed cell, adult stem cells, particularly stem cells derived from adipose tissue, appear to be more suitable than fibroblasts, neuronal stem cells and ES cells. For the scaffold, scaffolds formed from natural components are more advantageous than scaffolds composed of artificial and synthetic materials. However, the blending of natural and synthetic materials may reduce the disadvantages of using synthetic material while also avoiding the disadvantages of using solely natural components. The growth factor is important as it enhances the repairing effect, particularly when the virus carrier used to transfect the stem cells enables the consistent expression of the growth factor gene. We hypothesize that the use of a combination of growth factors may be more effective than the use of a single growth factor. Therefore, the construction of a virus capable of carrying several genes together requires further study. In conclusion, although tissue engineering has a promising future for the treatment of SCI, extensive further studies are necessary for the successful treatment of SCI to be achieved.

\section{Acknowledgements}

The authors are grateful to Ying Mao for the guidance provided while writing this review.

\section{References}

1. Findley PA, Banerjea R and Sambamoorthi U: Excess mortality associated with mental illness and substance use disorders among veteran clinic users with spinal cord injury. Disabil Rehabil 33: 1608-1615, 2011.

2. Wang M, Zhai P, Chen X, Schreyer DJ, Sun X and Cui F: Bioengineered scaffolds for spinal cord repair. Tissue Eng Part B Rev 17: 177-194, 2011

3. Hulsebosch CE: Recent advances in pathophysiology and treatment of spinal cord injury. Adv Physiol Educ 26: 238-255, 2002.

4. Baumgaertner W, Spitzbarth I and Beineke A: New knowledge on the pathology of spinal cord disease in dogs - views on therapy. Praktische Tierarzt 93: 794-796, 2012.

5. Wasner G: Spinal cord injury pain - from symptom to pathology. In: Proceedings of the 3rd International Congress on Neuropathic Pain (NeuPSIG). Medimond, Bologna, pp107-111, 2010.

6. Shimizu H, Kakita A and Takahashi H: Spinal cord tau pathology in cervical spondylotic myelopathy. Acta Neuropathol 115: 185-192, 2008

7. Norenberg MD, Smith J and Marcillo A: The pathology of human spinal cord injury: defining the problems. J Neurotrauma 21: 429-440, 2004.

8. Banerjea R, Findley PA, Smith B, Findley $T$ and Sambamoorthi U: Co-occurring medical and mental illness and substance use disorders among veteran clinic users with spinal cord injury patients with complexities. Spinal Cord 47: 789-795, 2009.

9. May L, Day R and Warren S: Evaluation of patient education in spinal cord injury rehabilitation: knowledge, problem-solving and perceived importance. Disabil Rehabil 28: 405-413, 2006.

10. Cruz CD and Cruz F: Spinal cord injury and bladder dysfunction: new ideas about an old problem. ScientificWorldJournal 11: 214-234, 2011.

11. Qu WS, Tian DS, GUO ZB, et al: Inhibition of EGFR/MAPK signaling reduces microglial inflammatory response and the associated secondary damage in rats after spinal cord injury. J Neuroinflammation 9: 178, 2012.

12. Madigan NN, McMahon S, O'Brien T, Yaszemski MJ and Windebank AJ: Current tissue engineering and novel therapeutic approaches to axonal regeneration following spinal cord injury using polymer scaffolds. Respir Physiol Neurobiol 169: 183-199, 2009.

13. Hernandez J, Torres-Espín A and Navarro X: Adult stem cell transplants for spinal cord injury repair: current state in preclinical research. Curr Stem Cell Res Ther 6: 273-287, 2011.

14. He X, Fu W and Zheng J: Cell sources for trachea tissue engineering: past, present and future. Regen Med 7: 851-863, 2012.

15. Malik A and Khan W: Stem cell therapy and tissue engineering applications for bone. Curr Stem Cell Res Ther 8: 183-184, 2013.

16. Khan WS and Malik A: Stem cell therapy and tissue engineering applications for cartilage regeneration. Curr Stem Cell Res Ther 7: 241-242, 2012.

17. Salehi M, Pasbakhsh P, Soleimani M, et al: Repair of spinal cord injury by co-transplantation of embryonic stem cell-derived motor neuron and olfactory ensheathing cell. Iran Biomed J 13: 125-135, 2009.

18. Wilcock AC, Swedlow JR and Storey KG: Mitotic spindle orientation distinguishes stem cell and terminal modes of neuron production in the early spinal cord. Development 134: 1943-1954, 2007. 
19. Daadi MM, Grueter BA, Malenka RC, Redmond DE Jr and Steinberg GK: Dopaminergic neurons from midbrain-specified human embryonic stem cell-derived neural stem cells engrafted in a monkey model of Parkinson's disease. PLoS One 7: e41120, 2012.

20. Meamar R, Dehghani L and Karamali F: Toxicity effects of methamphetamine on embryonic stem cell-derived neuron. J Res Med Sci 17: 470-474, 2012.

21. Tavares I: Human neural stem cell transplantation in spinal cord injury models: how far from clinical application? Stem Cell Res Ther 4: 61, 2013.

22. Deda H, Inci MC, Kurekci AE, et al: Treatment of chronic spinal cord injured patients with autologous bone marrow-derived hematopoietic stem cell transplantation: 1-year follow-up. Cytotherapy 10: 565-574, 2008.

23. Kim JW, Ha KY, Molon JN and Kim YH: Bone marrow-derived mesenchymal stem cell transplantation for chronic spinal cord injury in rats: comparative study between intralesional and intravenous transplantation. Spine (Phila Pa 1976) 38: E1065-E1074, 2013.

24. Alessandri G, Pagano S, Bez A, et al: Isolation and culture of human muscle-derived stem cells able to differentiate into myogenic and neurogenic cell lineages. Lancet 364: 1872-1883, 2004.

25. Lavasani M, Lu A, Thompson SD, Robbins PD, Huard J and Niedernhofer LJ: Isolation of muscle-derived stem/progenitor cells based on adhesion characteristics to collagen-coated surfaces. Methods Mol Biol 976: 53-65, 2013.

26. Wu X, Wang S, Chen B and An X: Muscle-derived stem cells: isolation, characterization, differentiation, and application in cell and gene therapy. Cell Tissue Res 340: 549-567, 2010.

27. Danisovic L, Varga I, Polák S, Bajciková B, Adamkov M and Vojtassák J: Biological and morphological characterization of in vitro expanded human muscle-derived stem cells. Tsitologiia 53: 482-487, 2011.

28. Danisovic L, Varga I, Polák S, Ulicna M, Bohmer D and Vojtassák J: Morphology of in vitro expanded human muscle-derived stem cells. Biomed Pap Med Fac Univ Palacky Olomouc Czech Repub 152: 235-238, 2008.

29. Woo JC, Bae WJ, Kim SJ, et al: Transplantation of muscle-derived stem cells into the corpus cavernosum restores erectile function in a rat model of cavernous nerve injury. Korean J Urol 52 359-363, 2011.

30. Stulpinas A, Imbrasaité A and Kalvelyté AV: Daunorubicin induces cell death via activation of apoptotic signalling pathway and inactivation of survival pathway in muscle-derived stem cells. Cell Biol Toxicol 28: 103-114, 2012.

31. Shibuya M, Miura T, Fukagawa Y, et al: Tongue muscle-derived stem cells express connexin 43 and improve cardiac remodeling and survival after myocardial infarction in mice. Circ J 74 1219-1226, 2010

32. Kwon EB, Lee JY, Piao S, Kim IG, Ra JC and Lee JY: Comparison of human muscle-derived stem cells and human adipose-derived stem cells in neurogenic trans-differentiation. Korean J Urol 52: 852-857, 2011

33. Kwon JS, Kim GH, Kim da Y, et al: Chitosan-based hydrogels to induce neuronal differentiation of rat muscle-derived stem cells Int J Biol Macromol 51: 974-979, 2012.

34. Zuk PA: The adipose-derived stem cell: looking back and looking ahead. Mol Biol Cell 21: 1783-1787, 2010.

35. Wilson B, Liotta LA and Petricoin EF: Dynamic protein pathway activation mapping of adipose-derived stem cell differentiation implicates novel regulators of adipocyte differentiation. Mol Cell Proteomics 12: 2522-2535, 2013.

36. Su SJ, Chang KL, Su SH, Yeh YT, Shyu HW and Chen KM Caffeine regulates osteogenic differentiation and mineralization of primary adipose-derived stem cells and a bone marrow stromal cell line. Int J Food Sci Nutr 64: 429-436, 2013.

37. Merceron C, Portron S, Masson M, et al: The effect of two- and three-dimensional cell culture on the chondrogenic potential of human adipose-derived mesenchymal stem cells after subcutaneous transplantation with an injectable hydrogel. Cell Transplant 20: 1575-1588, 2011.

38. Liqing Y, Jia G, Jiqing C, et al: Directed differentiation of motor neuron cell-like cells from human adipose-derived stem cells in vitro. Neuroreport 22: 370-373, 2011.

39. Valenzuela CD, Allori AC, Reformat DD, et al: Characterization of adipose-derived mesenchymal stem cell combinations for vascularized bone engineering. Tissue Eng Part A 19: 1373-1385, 2013.
40. Kleintjes WG: Treatment of basal cell carcinoma with autogenous growth factors and adipose-derived stem cells. Plast Reconstr Surg 126: 312e-313e, 2010.

41. Sterodimas A, de Faria J, Nicaretta B and Pitanguy I: Tissue engineering with adipose-derived stem cells (ADSCs): current and future applications. J Plast Reconstr Aesthet Surg 63: 1886-1892, 2010

42. Oh JS, Park IS, Kim KN, Yoon do H, Kim SH and $\mathrm{Ha} Y$ Transplantation of an adipose stem cell cluster in a spinal cord injury. Neuroreport 23: 277-282, 2012

43. Barriga A, Medrano M, De-Juan J and Burgos J: Intravenous infusion of adult adipose tissue stem cells for repairing spinal cord ischaemic lesions. An experimental study on animals. Rev Esp Cir Ortop Traumatol 57: 89-94, 2013 (In Spanish).

44. Ferrero-Gutierrez A, Menendez-Menendez Y, Alvarez-Viejo M, Meana A and Otero J: New serum-derived albumin scaffold seeded with adipose-derived stem cells and olfactory ensheathing cells used to treat spinal cord injured rats. Histol Histopathol 28: 89-100, 2013

45. Chung JY, Kim W, Im W, et al: Neuroprotective effects of adipose-derived stem cells against ischemic neuronal damage in the rabbit spinal cord. J Neurol Sci 317: 40-46, 2012.

46. Barry DS, Pakan JM, O'Keeffe GW and McDermott KW: The spatial and temporal arrangement of the radial glial scaffold suggests a role in axon tract formation in the developing spinal cord. J Anat 222: 203-213, 2013.

47. Roberts A, Li WC and Soffe SR: A functional scaffold of CNS neurons for the vertebrates: the developing Xenopus laevis spinal cord. Dev Neurobiol 72: 575-584, 2012.

48. Yoshii S, Ito S, Shima M, Taniguchi A and Akagi M: Functional restoration of rabbit spinal cord using collagen-filament scaffold. J Tissue Eng Regen Med 3: 19-25, 2009.

49. Ellis-Behnke RG and Schneider GE: Peptide amphiphiles and porous biodegradable scaffolds for tissue regeneration in the brain and spinal cord. Methods Mol Biol 726: 259-281, 2011.

50. Stokols S and Tuszynski MH: Freeze-dried agarose scaffolds with uniaxial channels stimulate and guide linear axonal growth following spinal cord injury. Biomaterials 27: 443-451, 2006.

51. Guo SZ, Ren XJ, Wu B and Jiang T: Preparation of the acellular scaffold of the spinal cord and the study of biocompatibility. Spinal Cord 48: 576-581, 2010.

52. Silva NA, Salgado AJ, Sousa RA, et al: Development and characterization of a novel hybrid tissue engineering-based scaffold for spinal cord injury repair. Tissue Eng Part A 16: 45-54, 2010.

53. Chung TW and Chang YL: Silk fibroin/chitosan-hyaluronic acid versus silk fibroin scaffolds for tissue engineering: promoting cell proliferations in vitro. J Mater Sci Mater Med 21: 1343-1351, 2010

54. Hilmi AB, Halim AS, Hassan A, Lim CK, Noorsal K and Zainol I: In vitro characterization of a chitosan skin regenerating template as a scaffold for cells cultivation. Springerplus 2: 79 , 2013.

55. Guan L, Tian P, Ge H, et al: Chitosan-functionalized silk fibroin 3D scaffold for keratocyte culture. J Mol Histol: May 1, 2013 (Epub ahead of print)

56. She Z, Liu W and Feng Q: Self-assembly model, hepatocytes attachment and inflammatory response for silk fibroin/chitosan scaffolds. Biomed Mater 4: 045014, 2009.

57. She Z, Jin C, Huang Z, Zhang B, Feng Q and Xu Y: Silk fibroin/chitosan scaffold: preparation, characterization, and culture with HepG2 cell. J Mater Sci Mater Med 19: 3545-3553, 2008.

58. Cigognini D, Satta A, Colleoni B, et al: Evaluation of early and late effects into the acute spinal cord injury of an injectable functionalized self-assembling scaffold. PLoS One 6: e19782, 2011.

59. Comolli N, Neuhuber B, Fischer I and Lowman A: In vitro analysis of PNIPAAm-PEG, a novel, injectable scaffold for spinal cord repair. Acta Biomater 5: 1046-1055, 2009.

60. Kubinová S, Horák D, Hejčl A, et al: SIKVAV-modified highly superporous PHEMA scaffolds with oriented pores for spinal cord injury repair. J Tissue Eng Regen Med: February 11, 2013 (Epub ahead of print).

61. Kang KN, Lee JY, Kim da Y, et al: Regeneration of completely transected spinal cord using scaffold of poly(D,L-lactide-coglycolide)/small intestinal submucosa seeded with rat bone marrow stem cells. Tissue Eng Part A 17: 2143-2152, 2011.

62. Liu T, Houle JD, Xu J, Chan BP and Chew SY: Nanofibrous collagen nerve conduits for spinal cord repair. Tissue Eng Part A 18: 1057-1066, 2012

63. Zhu Y, Wang A, Shen W, et al: Nanofibrous patches for spinal cord regeneration. Adv Funct Mater 20: 1433-1440, 2010. 
64. Teng YD, Lavik EB, Qu X, et al: Functional recovery following traumatic spinal cord injury mediated by a unique polymer scaffold seeded with neural stem cells. Proc Natl Acad Sci USA 99: 3024-3029, 2002.

65. Du BL, Zeng CG, Zhang W, Quan DP, Ling EA and Zeng YS: A comparative study of gelatin sponge scaffolds and PLGA scaffolds transplanted to completely transected spinal cord of rat. J Biomed Mater Res A: June 15, 2013 (Epub ahead of print).

66. McCall J, Weidner N and Blesch A: Neurotrophic factors in combinatorial approaches for spinal cord regeneration. Cell Tissue Res 349: 27-37, 2012.

67. de Leon RD: Could neurotrophins replace treadmill training as locomotor therapy following spinal cord injury? Focus on 'neurotrophic factors promote and enhance locomotor recovery in untrained spinalized cats'. J Neurophysiol 98: 1845-1846, 2007.

68. Sharma HS: Neurotrophic factors in combination: a possible new therapeutic strategy to influence pathophysiology of spinal cord injury and repair mechanisms. Curr Pharm Des 13: 1841-1874, 2007.

69. Sharma HS and Sharma A: Rodent spinal cord injury model and application of neurotrophic factors for neuroprotection. Methods Mol Biol 846: 393-415, 2012

70. Berry A, Bindocci E and Alleva E: NGF, brain and behavioral plasticity. Neural Plast 2012: 784040, 2012.

71. Allen SJ, Watson JJ, Shoemark DK, Barua NU and Patel NK: GDNF, NGF and BDNF as therapeutic options for neurodegeneration. Pharmacol Ther 138: 155-175, 2013.

72. Weishaupt N, Blesch A and Fouad K: BDNF: the career of a multifaceted neurotrophin in spinal cord injury. Exp Neurol 238: 254-264, 2012

73. Weishaupt N, Li S, Di Pardo A, Sipione S and Fouad K: Synergistic effects of BDNF and rehabilitative training on recovery after cervical spinal cord injury. Behav Brain Res 239: 31-42, 2013.

74. Mantilla CB, Gransee HM, Zhan WZ and Sieck GC: Motoneuron $\mathrm{BDNF} /$ TrkB signaling enhances functional recovery after cervical spinal cord injury. Exp Neurol 247: 101-109, 2013.

75. Stokols S, Sakamoto J, Breckon C, Holt T, Weiss J and Tuszynski MH: Templated agarose scaffolds support linear axonal regeneration. Tissue Eng 12: 2777-2787, 2006.

76. Shang AJ, Hong SQ, Xu Q, et al: NT-3-secreting human umbilical cord mesenchymal stromal cell transplantation for the treatment of acute spinal cord injury in rats. Brain Res 1391: 102-113, 2011.

77. Shumsky JS, Tobias CA, Tumolo M, Long WD, Giszter SF and Murray M: Delayed transplantation of fibroblasts genetically modified to secrete BDNF and NT-3 into a spinal cord injury site is associated with limited recovery of function. Exp Neurol 184: 114-130, 2003
78. Chen Q, Smith GM and Shine HD: Immune activation is required for NT-3-induced axonal plasticity in chronic spinal cord injury. Exp Neurol 209: 497-509, 2008.

79. Guo JS, Zeng YS, Li HB, et al: Cotransplant of neural stem cells and NT-3 gene modified Schwann cells promote the recovery of transected spinal cord injury. Spinal Cord 45: 15-24, 2007.

80. Wang X, Li Y, Gao Y, et al: Combined use of spinal cord-mimicking partition type scaffold architecture and neurotrophin-3 for surgical repair of completely transected spinal cord in rats. J Biomater Sci Polym Ed 24: 927-939, 2013.

81. Hara T, Fukumitsu H, Soumiya H, Furukawa Y and Furukawa S: Injury-induced accumulation of glial cell linederived neurotrophic factor in the rostral part of the injured rat spinal cord. Int J Mol Sci 13: 13484-13500, 2012.

82. Lin S, Wang Y, Zhang C and Xu J: Modification of the neurotrophin-3 gene promotes cholinergic neuronal differentiation and survival of neural stem cells derived from rat embryonic spinal cord in vitro and in vivo. J Int Med Res 40: 1449-1458, 2012

83. Yao L, Yao S, Daly W, Hendry W, Windebank A and Pandit A: Non-viral gene therapy for spinal cord regeneration. Drug Discov Today 17: 998-1005, 2012.

84. Bo X, Wu D, Yeh J and Zhang Y: Gene therapy approaches for neuroprotection and axonal regeneration after spinal cord and spinal root injury. Curr Gene Ther 11: 101-115, 2011.

85. Blesch A, Fischer I and Tuszynski MH: Gene therapy, neurotrophic factors and spinal cord regeneration. Handb Clin Neurol 109: 563-574, 2012.

86. Foust KD, Flotte TR, Reier PJ and Mandel RJ: Recombinant adeno-associated virus-mediated global anterograde delivery of glial cell line-derived neurotrophic factor to the spinal cord: comparison of rubrospinal and corticospinal tracts in the rat. Hum Gene Ther 19: 71-82, 2008.

87. Koda M, Hashimoto M, Murakami M, et al: Adenovirus vector-mediated in vivo gene transfer of brain-derived neurotrophic factor (BDNF) promotes rubrospinal axonal regeneration and functional recovery after complete transection of the adult rat spinal cord. J Neurotrauma 21: 329-337, 2004.

88. Blits B, Kitay BM, Farahvar A, Caperton CV, Dietrich WD and Bunge MB: Lentiviral vector-mediated transduction of neural progenitor cells before implantation into injured spinal cord and brain to detect their migration, deliver neurotrophic factors and repair tissue. Restor Neurol Neurosci 23: 313-324, 2005.

89. Morizono K and Chen IS: Targeted gene delivery by intravenous injection of retroviral vectors. Cell Cycle 4: 854-856, 2005. 